\title{
Rapid urinary antigen test for diagnosis of pneumococcal community-acquired pneumonia in adults
}

\author{
M.A. Marcos*, M.T. Jiménez de Anta*, J.P. de la Bellacasa*, J. González*, E. Martínez\#, \\ E. García\#, J. Mensa\#, A. de Roux", A. Torres"
}

Rapid urinary antigen test for diagnosis of pneumococcal community-acquired pneumonia in adults. M.A. Marcos, M.T. Jiménez de Anta, J.P. de la Bellacasa, J. González, E. Martínez, E. García, J. Mensa, A. de Roux, A. Torres. C) ERS Journals Ltd 2003.

ABSTRACT: Streptococcus pneumoniae is suspected to cause an important proportion of community-acquired pneumonia (CAP) whose aetiology cannot be detected with conventional tests.

In this study, the authors evaluated the diagnostic yield of a new immunochromatographic membrane test (ICT) for the detection of the $S$. pneumoniae antigen in the urine of patients admitted with diagnosed CAP. ICT was performed in unconcentrated and concentrated urine from all the patients. ICT was repeated 1 month after discharge in a group initially testing positive. The authors also studied the ICT in clinically stable human immunodeficiency virus type 1 (HIV1)-infected patients.

$S$. pneumoniae antigen was detected in all of the $68(100 \%)$ patients tested with definitive pneumococcal pneumonia. In five of these cases ICT was only positive when it had been performed on the patients. The $S$. pneumoniae antigen was also detected in $36(69.2 \%)$ of 52 patients with probable pneumococcal pneumonia and in 50 of 277 $(18 \%)$ patients without pneumococcal pneumonia. ICT remained positive in $16(69.5 \%)$ of 23 patients, 1 month after hospital discharge. Nasopharyngeal colonisation with S. pneumoniae was detected in $8(12 \%)$ of 68 clinically stable HIV1 infected patients, but none tested ICT positive.

The Binax NOW $\mathbb{R}$ immunochromatographic membrane test is a rapid, sensitive and specific test for detecting pneumococcal community-acquired pneumonia in adults. The test may remain positive for several weeks after pneumococcal pneumonia.

Eur Respir J 2003; 21: 209-214.
*Dept of Microbiology and ${ }^{*}$ Dept of
Infectious Diseases, Institute of Infec-
tions and Immunology, and "Dept of
Pneumology, Institute of Pneumology
and Thoracic Surgery, Hospital Clínic,
Institut d'Investigacions Biomèdiques
August Pi i Sunyer (IDIBAPS), Uni-
versitat de Barcelona, Barcelona, Spain.

Correspondence: A. Torres, Institute of Pneumology and Thoracic Surgery, Hospital Clínic Universitari, C/Villarroel 170, 08036 Barcelona, Spain.

Fax: 34932279813

E-mail: atorres@medicina.ub.es

Keywords: Community-acquired pneumonia, rapid diagnosis, Streptococcus pneumoniae, urinary antigen

Received: July 52002

Accepted after revision: October 25 2002

This work was supported by grants from the following: Sociedad Española de Neumología y Cirugía Torácica (SEPAR), Spanish Ministry of Health (FIS 00/0505), FUCAP (Maria Ravá), and Binax Inc (Portland, ME, USA).
Community-acquired pneumonia (CAP) is a common disease, representing the most frequent cause of hospital admission and mortality of infectious origin in developed countries [1]. Streptococcus pneumoniae is the leading cause of CAP $[1,2]$ and is responsible for $30-40 \%$ of CAP where the aetiology can be established following a routine diagnostic work-up. However, even in well-designed prospective studies, the aetiology of CAP remains elusive in $\sim 50 \%$ of CAP cases. Recent studies have suggested that S. pneumoniae may be underdiagnosed and that it may be responsible for at least one of three episodes of CAP without aetiological diagnosis [3, 4]. The lack of rapid and sensitive diagnostic methods for determining pneumococcal aetiology, the high mortality associated with pneumococcal CAP and the emerging antibiotic resistance in strains of $S$. pneumoniae, justify the need to improve the diagnosis of potential pneumococcal aetiology of CAP [5-7].

In a clinical setting, there are a number of obstacles to establish the pneumococcal aetiology with conventional diagnostic methods in patients with CAP. The isolation of S. pneumoniae from blood or pleural fluid, the gold standard test, is positive in only $15-30 \%$ of cases [8]. Diagnosis based only on sputum culture is controversial due to both nasopharyngeal carriage of pneumococci in healthy individuals and inadequate sputum specimens $[9,10]$. In addition, $\sim 30 \%$ of patients with CAP have been treated with antibiotics before admission, which may decrease the sensitivity of conventional methods [11]. Invasive specimens, such as those obtained by bronchoalveolar lavage (BAL), bronchoscopically retrieved protected specimen or transthoracic needle aspiration are generally considered to be the most reliable respiratory samples for determining the aetiology of pneumonia. Isolation of pneumococci from these specimens is considered proof of pneumococcal origin. However, invasive techniques are not routinely used because they require specialist training and may have side-effects [12, 13].

Rapid and sensitive microbiological methods providing a specific aetiological diagnosis, irrespective of prior antibiotic therapy, are subsequently required [14]. The use of polymerase chain reaction has been 
evaluated to improve the diagnostic yield of pneumococcal aetiology in patients with CAP [15-17]. However, this technique is complex and time-consuming and has not yet been fully standardised. Antigen detection tests have also been used for many years in the diagnosis of pneumococcal pneumonia; however, the diagnostic yield varies widely depending on the body fluid studied, the technique used and the bacterial antigen assessed [18-20].

An immunochromatographic membrane assay (ICT) has recently become available to detect the pneumococcal antigen in the urine of patients with pneumonia (Binax NOW $®$ S. pneumoniae Urinary Antigen Test; Binax, Portland, ME, USA). In contrast to other less sensitive bacterial antigen-tests, which detect capsular antigens, ICT detects the $S$. pneumoniae C-polysaccharide which is found in the cell wall and is common to all serotypes [21]. In this study the sensitivity and the specificity of the ICT for its clinical use in the diagnosis of pneumococcal aetiology in patients admitted for CAP was evaluated. In addition the specificity of the ICT in a group of clinically stable human immunodeficiency virus (HIV) positive patients was also assessed.

\section{Methods}

\section{Design}

The microbiological aetiology of CAP was prospectively assessed using an intensive diagnostic approach with conventional laboratory techniques and ICT. As the sensitivity of the conventional microbiological methods for diagnosing pneumococcal CAP is limited, the clinical interpretation of a positive ICT without a positive culture from the patient with CAP may be difficult. The result could either be a false positive, due to a lack of specificity of the new test or a true positive result not properly diagnosed with conventional methods. To further discriminate the specificity of ICT for the diagnosis of pneumococcal CAP, the performance of the ICT in two different settings was investigated. One consisted of a subgroup of patients who were both culture proven and ICT positive 1 month after pneumococcal CAP diagnosis. The second group included HIV type 1 (HIV1)-infected patients because of their high risk of developing nasopharyngeal colonisation with $S$. pneumoniae.

\section{Patients}

Patients with community-acquired pneumonia. Between January 2000-April 2001, consecutive adults ( $\geqslant 18$ yrs) diagnosed with CAP and admitted to Hospital Clinic Institut d'Investigacions Biomèdiques August Pi i Sunyer (Universitat de Barcelona, Barcelona, Spain) were prospectively studied. CAP was defined as the presence of symptoms of a lower respiratory tract infection, combined with a new infiltrate on chest radiography, no emerging alternative diagnosis during follow-up and nonhospitalisation of the patient in the preceding $72 \mathrm{~h}$.
Clinical, radiographic, and laboratory data were recorded and entered into a computer database as described elsewhere [2, 5].

Microbiological evaluation included routine sampling of sputum, blood and urine at admission. All sputum samples were Gram-stained, but only those from the lower respiratory tract were subsequently cultured. A good quality sputum sample required the presence of $>25$ polymorphonuclear leukocytes and $<10$ squamous epithelial cells $\times 100$ field [22]. When clinically considered, pleural puncture, tracheobronchial aspiration (TBAS), or BAL were also used to obtain samples for Gram-staining and culture. Respiratory samples were cultured on sheep blood, chocolate, and Sabouraud agar. Results of quantitative cultures were expressed as colony-forming units per $\mathrm{mL}\left(\mathrm{cfu} \cdot \mathrm{mL}^{-1}\right)$. A total of two blood samples were aerobically and anaerobically cultured (Bactec 9240; Becton Dickinson, Shannon, Ireland). Urine samples were obtained for the detection of the Legionella pneumophila antigen (Biotest Legionella Urine Antigen EIA; Biotest, Dreieich, Germany) and for the $S$. pneumoniae antigen (Binax NOW $\mathrm{R}$. pneumoniae Urinary Antigen Test; Binax) as previously described $[23,24]$. The results disclosing the weak-intensity coloured line were considered as negative.

Only concentrated urine was assessed for the detection of L. pneumophila antigen, whereas both concentrated and unconcentrated urine were used for detection of the $S$. pneumoniae antigen. Urine samples were boiled for $5 \mathrm{~min}$ and centrifuged at $1,000 \times g$ for $15 \mathrm{~min}$. The urine was concentrated 25 -fold by selective ultrafiltration (Urifil-10 Concentrator; Millipore Corporation, Bedford, MA, USA) [25]. Serological tests to detect immunoglobulin (Ig)M against Chlamydia pneumoniae and Mycoplasma pneumoniae were performed at the admission stage. For the rest of the serological tests, serum samples obtained at admission and 4 weeks thereafter were used for paired serology. Complement fixation tests were performed for influenza viruses $A$ and $B$, parainfluenzae viruses 1,2 , and 3 , adenovirus and respiratory syncitial virus. A microimmunofluorescence method was used to detect antibodies against C. pneumoniae, Coxiella burnetii, Chlamydia psittaci, L. pneumophila and M. pneumoniae. An enzyme immunoassay was carried out for the detection IgM against $M$. pneumoniae.

The aetiology of CAP was classified as probable if a valid sputum sample yielded $\geqslant 1$ predominant bacterial strain. The aetiology was considered definitive if one of the following criteria was met: 1) blood or pleural fluid yielding a bacterial or fungal pathogen; 2) a four-fold increase in the $\mathrm{IgG}$ titre for $C$. pneumoniae, C. psittaci, L. pneumophila, M. pneumoniae, C. burnetii, and respiratory viruses, an initial titre $\geqslant 1: 256$ or a positive urine antigen for $L$. pneumophila, an IgMpositive titre for $M$. pneumoniae, or an increase in the IgM titre $(\geqslant 1: 32)$ for $C$. pneumoniae; 3$)$ bacterial growth in cultures of TBAS $\geqslant 10^{5} \mathrm{cfu} \cdot \mathrm{mL}^{-1}$, and in $\mathrm{BAL} \geqslant 10^{4} \mathrm{cfu} \cdot \mathrm{mL}^{-1}$. Growth of fungi in respiratory samples were considered diagnostically valid only in the presence of a concomitant positive blood culture growing the same microorganism. The finding of Pneumocystis carinii cysts with methenamine-silver 
Table 1. - Comparison of the conventional diagnostic methods and the Binax NOW $\mathbb{R}$ Streptococcus pneumoniae urinary antigen test in patients with CAP

\begin{tabular}{|c|c|c|c|c|c|c|}
\hline & \multirow{2}{*}{$\begin{array}{l}\text { Sputum } \\
\text { culture }\end{array}$} & \multirow{2}{*}{$\begin{array}{l}\text { Invasive } \\
\text { respiratory } \\
\text { samples }\end{array}$} & \multirow{2}{*}{$\begin{array}{l}\text { Blood } \\
\text { culture }\end{array}$} & \multirow{2}{*}{$\begin{array}{l}\text { Others } \\
\text { methods }\end{array}$} & \multicolumn{2}{|c|}{ Urinary antigen test } \\
\hline & & & & & $\mathrm{NCU}$ & $\mathrm{CU}$ \\
\hline \multicolumn{7}{|l|}{ Pneumococcal pneumonia } \\
\hline \multicolumn{7}{|l|}{ Definitive $(n=68)$} \\
\hline Blood culture & 0 & 0 & 28 & 0 & 23 & 28 \\
\hline Blood culture and respiratory samples & 12 & 5 & 17 & 0 & 17 & 17 \\
\hline Respiratory samples & 0 & 23 & 0 & 0 & 12 & 23 \\
\hline Probable $(n=52)$ & 52 & 0 & 0 & 0 & 23 & 36 \\
\hline Nonpneumococcal pneumonia $(n=107)$ & 30 & 15 & 5 & 62 & 5 & 7 \\
\hline Pneumonia by an unidentified pathogen $(n=171)$ & 0 & 0 & 0 & 0 & 29 & 43 \\
\hline
\end{tabular}

NCU: nonconcentrate urine; CU: concentrate urine. ${ }^{\#}$ : tracheobronchial aspiration, bronchoalveolar lavage, pleural puncture; ${ }^{\circ}$ : Legionella pneumophila antigen, serology.

stain in the BAL was considered as positive diagnostic of $P$. carinii pneumonia.

Asymptomatic patients with recent pneumococcal community-acquired pneumonia. The ICT was repeated in concentrated urine 1 month after discharge in 23 patients with proven diagnosis of pneumococcal CAP by both conventional microbiological methods and ICT.

Asymptomatic human immunodeficiency type 1-infected patients. A total of 68 stable adult HIV1-infected patients, without symptoms of respiratory tract infection for the previous 4 weeks, were cross-sectionally studied. Nasopharynx swab specimens were cultured to detect $S$. pneumoniae colonisation. In addition, urine samples were concommitantly obtained and concentrated for ICT.

\section{Interpretation of study results}

The sensitivity and the specificity of ICT to diagnose the pneumococcal aetiology of CAP were calculated in the group of patients with CAP according to standard formulae. The results obtained with the ICT were compared with the results provided with conventional cultures. The specificity of the ICT was further assessed by studying its performance in two groups of patients without active infection but with a potential high risk of testing ICT positive, either due to a recent pneumococcal CAP or a high risk of pneumococcal nasopharyngeal colonisation, respectively.

\section{Results}

\section{Patients with community-acquired pneumonia}

A total of 398 patients (314 male, 79\%) with CAP were included $84(21 \%)$ of whom had HIV1 infection. The mean age was 50 yrs (range 24-95). The patients could be distributed into four groups of CAP: definitive pneumococcal CAP $(n=68)$, probable pneumococcal CAP $(n=52)$, nonpneumococcal CAP $(n=107)$, and CAP by an unidentified pathogen $(n=171)($ table 1$)$. Figure 1 summarises the outcome of the 398 patients with CAP according to ICT and culture results of blood and respiratory samples.
A good quality sputum was available from 183 $(46 \%)$ patients. Additional respiratory samples such as TBAS, pleural fluid or BAL were obtained from 48 $(12 \%), 20(5 \%)$, and $10(2.5 \%)$ patients, respectively. Blood cultures and detection of L. pneumophila and S. pneumoniae urinary antigens were performed on all of the patients. Serologies for atypical pathogens and respiratory viruses were obtained from 366 patients as single $(n=216)$ or paired samples $(n=150)$.

By using conventional laboratory techniques, an aetiological diagnosis was achieved in 227 (57\%) patients, of which $120(53 \%)$ were due to $S$. pneumoniae (table 2). S. pneumoniae was isolated from the blood cultures of 28 patients, from respiratory samples of 75 patients or both from 17 patients. S. pneumoniae was recovered from respiratory samples including sputum $(n=64)$, TBAS $(n=23)$, pleural fluid $(n=4)$ and BAL $(n=1)$.

The $S$. pneumoniae antigen was detected in 109 nonconcentrated urine samples and in 154 concentrated urine samples. All patients with a positive ICT in nonconcentrated urine samples also tested positive in concentrated samples. S. pneumoniae antigen was detected in 68 of 68 patients with definitive pneumococcal pneumonia $(100 \%)$. In five patients with positive blood cultures, the ICT was positive only after concentrating the urine. From patients with a diagnosis of probable pneumococcal pneumonia, antigen was detected

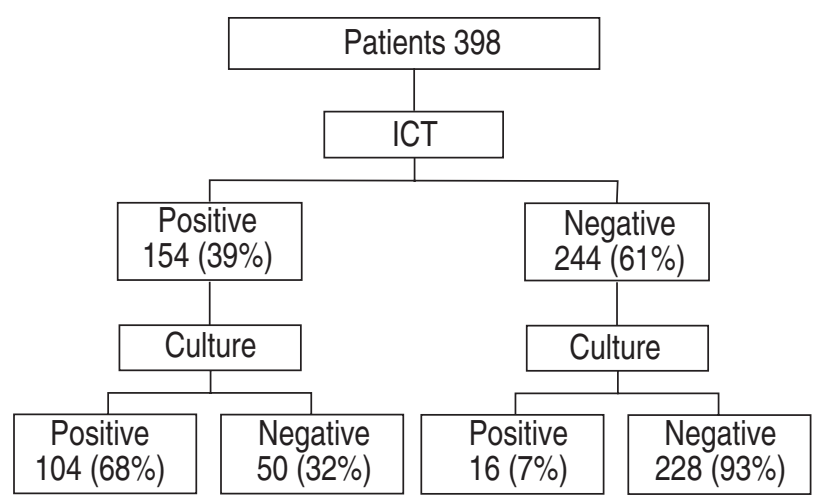

Fig. 1.- The number of patients with community-acquired pneumonia testing positive and negative on the immunochromatographic membrane test (ICT) and the results from the blood or respiratory cultures according to the results of the ICT. 
Table 2. - Aetiology of community-acquired pneumonia in 398 patients admitted using conventional laboratory diagnostic methods

\begin{tabular}{lcc}
\hline Microorganism & $\mathrm{n}$ & $\%$ \\
\hline Streptococcus pneumoniae & 112 & 49 \\
Legionella pneumophila & 17 & 7 \\
Haemophilus influenzae & 26 & 11 \\
Neisseria meningitidis & 1 & $<1$ \\
Moraxella catarrhalis & 7 & 3 \\
Staphylococcus aureus & 7 & 3 \\
Enterobacter cloacae & 1 & $<1$ \\
Escherichia coli & 1 & $<1$ \\
Prevotella melaninogenica & 1 & $<1$ \\
Pseudomonas aeruginosa & 3 & 1 \\
Candida albicans & 1 & $<1$ \\
Pneumocystis carinii & 2 & 1 \\
Chlamydia pneumoniae & 4 & 2 \\
Mycoplasma pneumoniae & 9 & 4 \\
Viral agents & 25 & 11 \\
Mixed aetiology & & \\
S. pneumoniae, M. pneumoniae & 2 & 1 \\
S. pneumoniae, C. pneumoniae & 3 & 1 \\
S. pneumoniae, H. influenzae & 3 & 1 \\
H. influenzae, M. pneumoniae & 1 & $<1$ \\
H. influenzae, L. pneumophila & 1 & $<1$ \\
\hline
\end{tabular}

in 36 of 52 urine samples $(69.2 \%$; table 1$)$. In the 16 patients, who were ICT-negative, $S$. pneumoniae was isolated from the sputum but not from blood cultures. At least one copathogen was also isolated in seven of the 16 sputum samples (Pseudomonas aeruginosa, $\mathrm{n}=1$; Moraxella catarrhalis, $\mathrm{n}=1$; Haemophilus influenzae, $\mathrm{n}=3$; Enterobacter cloacae, $\mathrm{n}=1 ; P$. carinii, $\mathrm{n}=1$ ).

The overall specificity of ICT was $82 \%$. The specificity was $93 \%$ for nonpneumococcal CAP and $75 \%$ for CAP by an unidentified pathogen (table 1 ). When the group of 50 patients with negative cultures and positive ICT results were analysed, a causative microorganism was identified in seven of the patients by conventional methods, these were as follows; $M$. pneumoniae $(\mathrm{n}=1), C$. pneumoniae $(\mathrm{n}=1), P$. carinii $(\mathrm{n}=1), H$. influenzae $(\mathrm{n}=2)$, Escherichia coli $(\mathrm{n}=1)$ and influenza A virus $(n=1)$. A total of $21(42 \%)$ of 50 patients had received antibiotic therapy prior to sample collection compared with two $(1.6 \%)$ of the 120 patients in whom $S$. pneumoniae was isolated and tested positive for ICT $(\mathrm{p}<0.05$, Chi-squared test).

\section{Asymptomatic patients with recent pneumococcal community-acquired pneumonia}

The ICT was positive for $16(69.5 \%)$ of 23 patients, 1 month after pneumococcal CAP had been diagnosed (as defined by both conventional microbiological methods and ICT) despite satisfactory clinical, laboratory, and radiographic evolution.

Asymptomatic human immunodeficiency virus type 1-infected patients

S. pneumoniae was isolated from nasopharyngeal swab specimens from $8(12 \%)$ of the 68 asymptomatic
HIV1-infected patients and the ICT assay in urine was negative in all the patients.

\section{Discussion}

The most important findings from this study are: 1) $S$. pneumoniae continues to be the main aetiology of CAP; 2) the concentration of urine increases the yield of the ICT for pneumococcal CAP; 3) the ICT shows a good sensitivity and specificity for the diagnosis of pneumococcal CAP; and 4) true positive results of ICT may remain positive for several weeks.

In this prospective study, the cause of CAP was identified in $57 \%$ of the patients with the use of intensive conventional microbiological methods, with $S$. pneumoniae being the most common agent $(\mathrm{n}=120$, $53 \%$ ). The data agrees with previously published reports and highlights the potential importance of S. pneumoniae as a causative agent of CAP [1].

In contrast to good quality sputum, which can not always be spontaneously obtained, and to invasive respiratory samples, which are not routinely collected, urine may be easily collected from all patients with CAP. The ICT was performed in both concentrated and nonconcentrated urine because data in a similar test to detect L. pneumophila suggested that concentrated urine may have a higher diagnostic yield [25]. Urine concentration in this study did not represent additional work since this was also necessary to determine the urinary antigen of L. pneumophila. The positivity of ICT in the present study increased from $109(27 \%)$ nonconcentrated to $154(39 \%)$ concentrated urine samples. There was no conclusive data supporting urine concentration to be necessary for, or to improve ICT yield. However, the fact that five (11.1\%) episodes of bacteraemic pneumococcal CAP in the present study tested ICT positive, only after concentrating the urine, supports the necessity to concentrate the urine for this test. Similar findings were reported by Murdoch et al. [26]. The authors of that study described how four patients with positive blood cultures for $S$. pneumoniae had negative ICT results from nonconcentrated urine samples and positive ICT results were found for two of the patients in concentrated urine samples (no data regarding urine concentration were given for the remaining two patients). Ausina et al. [27] found the concentration of urine extremely useful to obtain optimal results on detecting capsular pneumococcal antigen by counterimmunoelectrophoresis. Dominguez et al. [23] also concluded that the concentration of urine improves the diagnosis of pneumococcal aetiology. The concentration of urine is a simple, rapid $(\leqslant 2 \mathrm{~h})$ procedure that can be easily performed, and in summary it seems reasonable to concentrate urine when using ICT to improve the sensitivity of this method.

The ICT in concentrated urine correctly identified 104 of 120 pneumococcal aetiologies diagnosed by culture. In the cases of pneumococcal CAP detected by culture but not with ICT $(\mathrm{n}=16), S$. pneumoniae was detected in only sputum but not in blood cultures. The value of sputum for the diagnosis of CAP, even in the setting of good quality criteria, remains controversial 
[9]. The potential role of $S$. pneumoniae as the cause of CAP in these cases is arguable due to the presence of a copathogen which may explain the aetiology of the CAP found in seven of the 16 sputum samples.

With the culture results as a reference, the overall specificity of the ICT to diagnose pneumococcal CAP in concentrated urine was $82 \%$. Several issues should be taken into account when analysing the meaning of positive ICT results in 50 patients with negative cultures. The reliance on microbial cultures, either from respiratory samples or from blood, for the diagnosis of pneumococal CAP may be jeopardised by the relatively low sensitivity of cultivation. This was reflected in the present study as the diagnostic yield from blood cultures of pneumococcal CAP was only $37.5 \%$. At least one respiratory sample could be obtained from all the patients, with sputum being the most commonly obtained, although with good quality criteria in only $47 \%$ of the patients. This finding, which has also been reported elsewhere [28], seriously limits the diagnostic yield of sputum for the aetiological diagnosis of CAP. In addition, $42 \%$ of the patients testing ICT-positive but with negative cultures had received antibiotics prior to sample collection compared with $2 \%$ of the patients testing ICTpositive with positive cultures $(p<0.05$, Chi-squared test). Similar discordant findings between ICT and cultures in patients with pneumococcal meningitis, who had received prior antibiotic therapy, had been reported previously by the authors [24]. In contrast to culture, the results of the ICT should not be affected by the administration of antibiotics. The administration of antimicrobial therapy prior to admission has been reported as a cause of false-negative results not only for CAP, but also for other invasive bacterial diseases [29]. Therefore, the limited sensitivity of culture as a conventional microbiological method to diagnose pneumococcal CAP may not determine if the ICT-positive results are really true or false. No additional diagnostic tests were performed in combination with the cultural technique that may have helped gain further insight into the meaning of discordant patients with positive ICT results and negative cultures. In summary, it is unclear as to whether cases testing ICT-positive and culturenegative were either false-positive results of the ICT test (thus representing patients without pneumococcal CAP) or false-negative results of the culture (thus representing underdiagnosed cases of pneumococcal CAP). Further data supporting the high specifity of ICT was obtained from the results of the study with asymptomatic HIV1-infected patients. Negative ICT results were found consistently in all the 68 patients studied, even in the eight patients with $S$. pneumoniae isolated in the nasopharynx. The findings of Murdoch et al. [26], who did not detect pneumococcal antigen in urine from 188 control adult patients, support the high specificity of the ICT in adults, and complement the findings of the present study. However, Dowell ET AL [30] found that the ICT was often positive in healthy children who were colonised with $S$. pneumoniae. Consequently, the ICT test might have different performance characteristics in adults than in children.
Although the long persistence of antigens in urine, several days after the onset of symptoms, may be useful to detect pneumococcal aetiology in patients with CAP irrespective of antibiotic therapy, the interpretation of a positive result in patients with prior pneumococcal invasive infections should be made with caution, since it may be unrelated to the present episode of CAP. In the present study, ICT in concentrated urine was positive in $70 \%$ of the patients with pneumococcal CAP proven by both culture and ICT 1 month after the diagnosis of CAP despite good clinical, laboratory and radiographic evolution. Therefore, further studies are needed to ascertain how long positive ICT results may persist after an episode of pneumococcal CAP in order to properly interpret the ICT test in clinical practice.

In summary, the immunochromatographic membrane test is a sensitive and specific method for the diagnosis of pneumococcal community-acquired pneumonia in adults. It may be used as a complementary tool to conventional methods given the advantages of it being both rapid and simple and it lacking any influence by the prior treatment of the patient with antibiotics. Based on the results of this study, the authors recommend immunochromatographic membrane tests be performed on concentrated urine to increase the test sensitivity, and caution should be taken when interpreting the results if the patient has undergone a recent episode of community-acquired pneumonia.

\section{References}

1. Brown PD, Lerner SA. Community-acquired pneumonia. Lancet 1998; 352: 1295-1302.

2. Ruiz M, Ewig S, Marcos MA, et al. Etiology of community-acquired pneumonia: Impact of age, comorbidity, and severity. Am J Respir Crit Care Med 1999; 160: 397-405.

3. Ruiz-Gonzalez A, Falguera M, Nogués A, RubioCaballero. Is Streptococcus pneumoniae the leading cause of pneumonia of unknown etiology? A microbiologic study of lung aspirates in consecutive patients with community-acquired pneumonia. $\mathrm{Am} \mathrm{J} \mathrm{Med}$ 1999; 106: 385-390.

4. Menendez R, Cordoba J, de la Cuadra P, et al. Value of the polymerase chain reaction assay in noninvasive respiratory samples for diagnosis of communityacquired pneumonia. Am J Respir Crit Care Med 1999; 159: 1868-1873.

5. Ewig S, Ruiz M, Torres A, et al. Pneumonia acquired in the community through drug-resistant Streptococcus pneumoniae. Am J Respir Crit Care Med 1999; 159: 1835-1842.

6. Heffelfinger JD, Dowell SF, Jorgensen JH, et al. Management of community-acquired pneumonia in the era of pneumococcal resistance: a report from the drug-resistant Streptococcus pneumoniae therapeutic working group. Arch Intern Med 2000; 160: 13991408.

7. Niederman MS. Guidelines for the management of community-acquired pneumonia: current recommendations and antibiotic selection issues. Med Clin North Am 2001; 85: 1493-1509. 
8. Burman LA, Trollfors B, Andersson B, et al. Diagnosis of pneumonia by cultures, bacterial and viral antigen detection tests, and serology with special reference to antibodies against pneumococcal antigens. J Infect Dis 1991; 163: 1087-1093.

9. Lentino JR, Lucks DA. Nonvalue of sputum culture in the management of lower respiratory tract infections. J Clin Microbiol 1987; 25: 758-762.

10. Fine MJ, Orloff JJ, Rihs JD, et al. Evaluation of housestaff physicians preparation and interpretation of sputum gram strains for community-acquired pneumonia. J Gen Intern Med 1991; 6: 189-198.

11. Marston BJ, Plouffe JF, File TM, et al. Incidence of community-acquired pneumonia requiring hospitalization: results of a population based active surveillance study in Ohio. Arch Intern Med 1997; 157: 17091718.

12. Khan FW, Jones JM. Diagnosing bacterial respiratory infections by bronchoalveolar lavage. $J$ Infect Dis 1987; 155: 862-869.

13. Ruiz-Gonzalez A, Nogues A, Falguera M, et al. Rapid detection of pneumococcal antigen in lung aspirates: comparison with culture and PCR technique. Respir Med 1997; 91: 201-206.

14. Ausina V. Rapid laboratory diagnostic methods in respiratory infections. Curr Opin Infect Dis 1989; 2: 541-546.

15. Rudolph KM, Parkinson AJ, Black CM, Mayer LW. Evaluation of polymerase chain reaction for diagnosis of pneumococcal pneumonia. J Clin Microbiol 1993; 31: 2661-2666.

16. Dagan R, Schriker O, Hazan I, et al. Prospective study to determine clinical relevance of detection of pneumococcal DNA in sera of children by PCR. $J$ Clin Microbiol 1998; 36: 669-673.

17. Lorente L, Falguera M, Nogués A, Ruiz Gonzalez A, Merino MT, Rubio Caballero M. Diagnosis of pneumococcal pneumonia by polymerase chain reaction (PCR) in whole blood: a prospective clinical study. Thorax 2000; 55: 133-137.

18. Holmberg H, Krook A. Comparison of enzyme-linked immunosorbent assay with coagglutination and latex agglutination for rapid diagnosis of pneumococcal pneumonia by detecting antigen in sputa. Eur J Clin Microbiol 1986; 5: 282-286.

19. Lenthe-Eboa S, Brighouse G, Auckenthaler R, et al. Comparison of immunological methods for diagnosis of pneumococcal pneumonia in biological fluids. Eur J Clin Microbiol 1987; 6: 28-34.
20. Garcia A, Rosón B, Perez JL, et al. Usefulness of PCR and antigen latex agglutination test with samples obtained by transthoracic needle aspiration for diagnosis of pneumococcal pneumonia. J Clin Microbiol 1999; 37: 709-714.

21. Jenning HJ, Lugowski C, Young NM. Structure of the complex polysaccharide C substance from Streptococcus pneumoniae type 1. Biochemistry 1980; 19: 4712-4719.

22. Barlett JG, Finegold SM. Bacteriology of expectorated sputum with quantitative culture and wash technique compared to transtracheal aspirates. Am Rev Respir Dis 1978; 117: 1019-1027.

23. Dominguez J, Gali N, Blanco S, et al. Detection of Streptococcus pneumoniae antigen by a rapid immunochromatographic assay in urine samples. Chest 2001; 119: 243-249.

24. Marcos MA, Martinez E, Almela M, Menzsa J, Jimenez de Anta MT. New rapid antigen test for diagnosis of pneumococcal meningitis. Lancet 2001; 357: 1499-1500.

25. Dominguez JA, Gali N, Pedroso P, et al. Comparison of the Binax legionella urinary antigen enzyme immunoassay (EIA) with the Biotest legionella urine antigen EIA for detection of legionella antigen in both concentrated and nonconcentrated urine samples. J Clin Microbiol 1998; 36: 2718-2722.

26. Murdoch DR, Laing RTR, Mills GD, et al. Evaluation of a rapid immunochromatographic test for detection of Streptococcus pneumoniae antigen in urine samples from adults with community-acquired pneumonia. J Clin Microbiol 2001; 39: 3495-3498.

27. Ausina V, Coll P, Sambeat M, et al. Prospective study on the etiology of community-acquired pneumonia in children and adults in Spain. Eur J Clin Microbiol Infect Dis 1988; 7: 343-347.

28. Rosón B, Carratala J, Verdaguer R, Dorca J, Manresa F, Gudiol F. Prospective study of the usefulness of sputum gram stain in the initial approach to community-acquired pneumonia requiring hospitalization. Clin Infect Dis 2000; 31: 869-874.

29. Cunha BA. Antibiotic treatment of sepsis. Med Clin North Am 1995; 79: 551-558.

30. Dowell SF, Garman RL, Liu G, Levine OS, Yang YH. Evaluation of Binax Now, an assay for the detection of pneumococcal antigen in urine samples, performed among pediatric patients. Clin Infect Dis 2001; 32: 824-825. 